\title{
Myelomatous involvement of the dura mater: a rare complication of multiple myeloma
}

\author{
P Roddie, D Collie, P Johnson
}

\begin{abstract}
A case of myelomatous involvement of the dura mater is reported. The patient presented with blurring of vision in the right visual field and left sided facial numbness. A magnetic resonance imaging scan of the head revealed extensive infiltration of the dura mater. Cerebrospinal fluid (CSF) analysis showed no plasmacytosis and although there was a raised CSF protein concentration, no paraprotein band was detected, despite the presence of serum paraprotein. The infiltration of the dura mater is likely to have arisen by spread from contiguous bone lesions, contrasting with the pattern of spread seen in myelomatous involvement of the leptomeninges, which probably occurs through haematogenous seeding of the meninges. Leptomeningeal involvement is associated with a very poor prognosis; however, this patient had a favourable response to combined chemotherapy and cranial radiotherapy, suggesting that myelomatous involvement of the dura mater should be considered as a distinct complication of myeloma.

(F Clin Pathol 2000;53:398-399)
\end{abstract}

Keywords: myeloma; dura mater; leptomeningeal

Involvement of the meninges is a very rare occurrence in multiple myeloma. This contrasts with other malignancies arising from cells of lymphoid origin, such as acute lymphoblastic leukaemia and non-Hodgkin's lymphoma, in which meningeal involvement is not infrequent. Dura mater involvement in multiple myeloma is even more rare, with only two previously reported cases. ${ }^{12}$ We report a case of dura mater involvement in myeloma, which was associated with florid neurological

Department of Haematology, Western General Hospital, Crewe Road, Edinburgh EH4 2XU, UK

P H Roddie

P R E Johnson

Department of Clinical Neurosciences,

Western General

Hospital

D Collie

Correspondence to:

Dr Roddie

email:

hroddie@holyrood.ed.ac.uk

Accepted for publication 20 October 1999 vey revealed diffuse osteopenia with lesions in the ribs. Biochemistry results were as follows: sodium, $144 \mathrm{mmol} /$ litre; potassium,
$5.8 \mathrm{mmol} / \mathrm{litre}$; urea, $17.0 \mathrm{mmol} / \mathrm{litre}$; and creatinine, $484 \mu \mathrm{mol} /$ litre. The concentration of $\beta_{2}$ microglobulin was $35.4 \mathrm{mg} /$ litre. Urine light chain excretion was $6 \mathrm{~g} / 24$ hours. Renal biopsy demonstrated myeloma cast nephropathy with advanced interstitial damage.

Treatment was started with ABCM (adriamycin, BCNU, cyclophosphamide, and melphalan) chemotherapy, but this was changed to weekly cyclophosphamide after one course because of prolonged cytopenias. The renal failure was managed conservatively. In May 1997, creatinine had fallen to $255 \mu \mathrm{g} /$ litre and urine light chain excretion was down to $0.72 \mathrm{~g} /$ 24 hours, with a serum $\kappa$ light chain paraprotein of $1 \mathrm{~g} /$ litre. A repeat trephine biopsy revealed a reduction in marrow cellularity to $10 \%$, but with continuing plasma cell infiltration. Weekly cyclophosphamide was continued until July 1997, then discontinued. The patient received monthly infusions of pamidronate, and from June 1997 was started on twice weekly subcutaneous erythropoietin as treatment for anaemia.

The patient remained well until June 1998 when bone pain recurred and her response to erythropoietin deteriorated. Two weeks later the patient developed blurring of vision in the right visual field and left sided facial numbness. Neurological examination revealed an inferior altitudinal field defect in the right eye, bilateral disc oedema, and left sided facial numbness in the region of the maxillary division of the fifth cranial nerve. A magnetic resonance imaging (MRI) scan of the head revealed extensive infiltration of the dura mater of the skull vault and dural reflections, with large lobulated masses over the skull falx cerebri and tentorium cerebelli (fig 1). A lumbar puncture was performed and demonstrated a raised cerebrospinal fluid (CSF) protein of $1.13 \mathrm{~g} /$ litre (normal range, 0.14-0.45), although immunofixation did not reveal a paraprotein band. No plasma cells were evident on CSF microscopy.

A single injection of intrathecal methotrexate was given at the same time as the diagnostic lumbar puncture. Systemic chemotherapy in the form of I-Dex (idarubicin and dexamethasone) was started immediately and continued for six cycles. The patient went on to receive seven fractions of palliative cranial radiotherapy. Symptomatically, her clinical condition improved, with resolution of the bone pain and an improvement in vision. At latest review, one year from the onset of neurological symptoms, visual acuity was 6/6 left eye and 9/6 right eye, with no demonstrable abnormality in visual field testing. In addition, the response to erythropoietin was restored, allowing the dose 


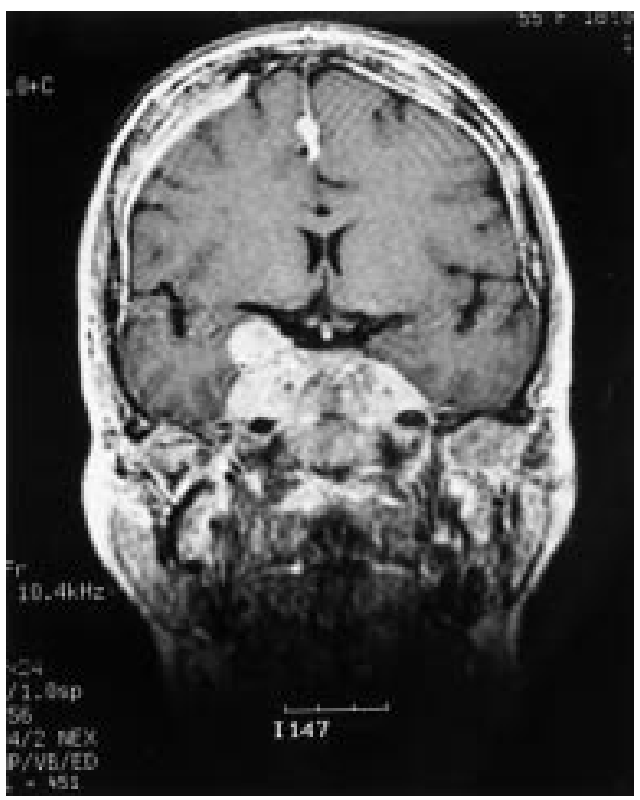

Figure 1 Post gadolinium enhanced T1 weighted magnetic resonance imaging (MRI) scan of brain. Coronal section showing diffuse undulating thickening of the calvarial dura with a further deposit in the falx.

to be reduced and then discontinued at six months, with no requirement for red blood cell transfusion. A repeat MRI scan six weeks after radiotherapy showed considerable improvement in the size of the dural masses.

\section{Discussion}

Meningeal involvement by myeloma is an uncommon event, with a recent review article citing only 28 previously reported cases in the literature. ${ }^{3}$ There have been only two previous reports of myelomatous involvement of the dura mater. ${ }^{12}$ In leptomeningeal involvement by myeloma, CSF plasmocytosis and/or an associated CSF paraprotein is the rule. The absence of these features in our case reflects the fact that the myelomatous involvement was limited to the dura mater, sparing the arachnoid, and therefore was not in direct contact with the CSF.

The mechanism of spread to the leptomeninges in myeloma is unknown, although it is thought to be comparable to that of leukaemic meningitis. Postmortem examination of children with acute lymphoblastic leukaemia has shown that leukaemic involvement of the central nervous system first becomes apparent in the walls of superficial arachnoid veins and surrounding adventitia. ${ }^{4}$ With more advanced stages of leukaemic infiltration, the arachnoid trabeculae are destroyed and leukaemic cells are able to spill over into the CSF, and are thus detectable on cytological examination. The mechanism of leptomeningeal spread in my- eloma is likely to take place by a related mechanism. Postmortem examinations of patients with meningeal myeloma have shown a similar histological pattern, with myeloma cells diffusely infiltrating the leptomeninges. ${ }^{5}$ The fact that in leukaemia and myeloma the initial stages of meningeal involvement affect the arachnoid veins suggests that haematogenous spread takes place from circulating leukaemia or myeloma cells. In the patient we describe, this is unlikely to be the case because the dura mater is a relatively avascular structure. It is more probable that spread took place from contiguous bone lesions, especially as it had been demonstrated that the patient had extensive skull involvement. Dural metastatic deposits most commonly arise from direct spread (particularly cranial vault) metastases. The tumour reaches the subdural space by breaching the inner table of the vault and fibrous dura. This is most commonly seen in adenocarcinomas such as breast and prostate. However, a rare form of pachymeningeal carcinomatosis is also recognised with discrete deposits in the meningeal reflections (falx and tentorium) distant form the bony skull. Presumably, these arise from dissemination along the subdural space because the leptomeninges are rarely affected. This latter form of disease is seen particularly with breast carcinoma and lymphoma.

The prognosis of myelomatous involvement of the meninges is extremely poor, with an average time from onset of neurological symptoms to death of eight weeks. ${ }^{3}$ This poor prognosis is in part explained by the fact that meningeal involvement generally occurs in patients with advanced disease, or with plasma cell leukaemia. Various treatments have been tried, including intrathecal chemotherapy, cranial irradiation, and systemic chemotherapy, but in general responses are partial and short lived.

The fact that the patient we describe had a favourable prognosis, and that the pattern of spread to the dura mater probably occurred from contiguous bone lesions as opposed to the haematogenous spread seen with involvement of the leptomeninges, suggest that involvement of the dura mater should be considered as a distinct complication of multiple myeloma.

1 Nomoto N, Saito H, Kashimura M, et al. Bence Jones type multiple myeloma showing diffuse infiltration to the dura mater by myeloma cells. Fapanese fournal of Clinical Hema-

2 Maldonado JE, Kyle RA, Ludwig J, et al. Meningeal Maldonado JE, Kyle RA, Ludwig J, et
myeloma. Arch Intern Med 1970;126:660-3.

3 Pizzuti P, Pertuiset E, Chaumonnot F, et al. Neuromeningeal sites of multiple myeloma: 3 cases and review of the literature. Rev Med Interne 1997;18:646-51.

4 Price RA, Johnson WW. The central nervous system in childhood leukemia. I. The arachnoid. Cancer 1973;31: 520-33.

5 Truong LD, Kim HS, Estrada R. Meningeal myeloma. Am f Clin Pathol 1982;78:532-5. 